\title{
Échapper à Shylock : la Hebrew Free Loan Association of Montreal entre antisémitisme et intégration, 1911-1913
}

\section{Sylvie Taschereau}

Volume 59, numéro 4, printemps 2006

URI : https://id.erudit.org/iderudit/013611ar

DOI : https://doi.org/10.7202/013611ar

Aller au sommaire du numéro

Éditeur(s)

Institut d'histoire de l'Amérique française

ISSN

0035-2357 (imprimé)

1492-1383 (numérique)

Découvrir la revue

Citer cet article

Taschereau, S. (2006). Échapper à Shylock : la Hebrew Free Loan Association of Montreal entre antisémitisme et intégration, 1911-1913. Revue d'histoire de l'Amérique française, 59(4), 451-480. https://doi.org/10.7202/013611ar
Résumé de l'article

En 1911, des représentants de l'élite juive montréalaise créent une société de prêts charitables. Lorsqu'ils en demandent l'incorporation aux autorités québécoises cependant, ils sont immédiatement confrontés à l'image du juif usurier. La fondation de la Hebrew Free Loan Association of Montreal nous entraîne au coeur des tensions que connaît la communauté juive au plus fort de l'immigration du début du $\mathrm{xx}^{\mathrm{e}}$ siècle. En même temps, les démarches de ses fondateurs et les réactions qu'elles provoquent révèlent la complexité des rapports qu'entretient cette minorité avec différentes composantes de la société québécoise à une époque où, au Québec comme ailleurs, l'antisémitisme se manifeste de plus en plus. Les efforts déployés pour obtenir l'incorporation de cet organisme et la lutte de représentations qui s'ensuit mettent en lumière aussi bien le pouvoir que la vulnérabilité de l'élite juive et l'importance de son rôle de médiation.
Tous droits réservés @ Institut d'histoire de l'Amérique française, 2006

Ce document est protégé par la loi sur le droit d'auteur. L'utilisation des services d'Érudit (y compris la reproduction) est assujettie à sa politique d'utilisation que vous pouvez consulter en ligne.

https://apropos.erudit.org/fr/usagers/politique-dutilisation/ 


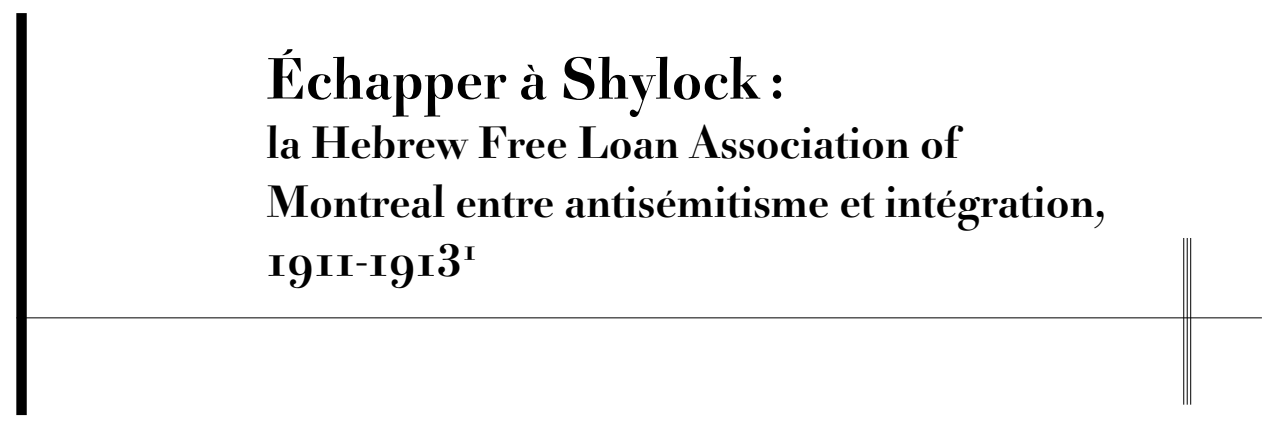

SYLVIE TASCHEREAU

Département des sciences humaines

Université du Québec à Trois-Rivières

RÉsumÉ • En 1911, des représentants de l'élite juive montréalaise créent une société de prêts charitables. Lorsqu'ils en demandent l'incorporation aux autorités québécoises cependant, ils sont immédiatement confrontés à l'image du juif usurier. La fondation de la Hebrew Free Loan Association of Montreal nous entraîne au cœur des tensions que connaît la communauté juive au plus fort de l'immigration du début du $x x^{\mathrm{e}}$ siècle. En même temps, les démarches de ses fondateurs et les réactions qu'elles provoquent révèlent la complexité des rapports qu'entretient cette minorité avec différentes composantes de la société québécoise à une époque où, au Québec comme ailleurs, l'antisémitisme se manifeste de plus en plus. Les efforts déployés pour obtenir l'incorporation de cet organisme et la lutte de représentations qui s'ensuit mettent en lumière aussi bien le pouvoir que la vulnérabilité de l'élite juive et l'importance de son rôle de médiation.

ABSTRACT - In the fall of 1911, representatives of the Montreal Jewish elite applied to Quebec authorities for the incorporation of a charitable institution that they had created,

1. L’analyse présentée ici s'inscrit dans une étude des rapports entre Juifs et non-Juifs qui se nouent à Montréal, dans la première moitié du $\mathrm{xx}^{\mathrm{e}}$ siècle, à travers les activités d'affaires. Cette recherche a été subventionnée par le CRSH entre 1998 et 2001. Outre les commentaires critiques des deux évaluateurs de la RHAF, ce texte a bénéficié de la lecture attentive et des suggestions de Bettina Bradbury ainsi que de celles de Pierre Anctil. Karine Hébert a bien voulu elle aussi en commenter une version précédente. Nous les remercions tous, de même que Janice Rosen, directrice des archives du Congrès juif du Canada, Eiran Harris, responsable des archives de la Bibliothèque publique juive de Montréal, et enfin Ève Lerner et Moishe Dolman, qui tous deux ont dépouillé pour nous la presse juive montréalaise. 
the Hebrew Free Loan Association of Montreal. They were immediately confronted by the stereotype of the Jewish loan shark. The founding of this society reveals some of the tensions experienced by the Montreal Jewish community during this peak period of mass migration at the beginning of the $20^{\text {th }}$ Century. The initiatives of its founders and the reactions they stirred up also reveal the complexity of the relationship that existed between the Jewish minority and various components of Quebec society at a time when anti-Semitism, in Quebec as elsewhere, was becoming more vocal. Their efforts to obtain the incorporation of the Hebrew Free Loan Association, as well as to reject the social identity that was being imposed on them, shed light on both the power and the vulnerability of the Jewish elite and on the crucial role it played in mediation.

\section{A L'automne de 1911, un groupe d'hommes d'affaires et de profession- Anels issus de l'élite de la communauté juive montréalaise entreprend} les démarches qui lui permettront de constituer officiellement en société la Hebrew Free Loan Association of Montreal' ${ }^{2}$ Cette institution, qui existe toujours aujourd'hui, devient dans les années suivantes et jusqu'aux lendemains de la Seconde Guerre mondiale l'une des plus prestigieuses de la communauté. Organisme à vocation charitable, c'est en même temps une institution financière puisqu'elle offre des prêts de montants modestes sans cependant exiger aucun intérêt. Mais dès les premières étapes de la procédure, la demande d'incorporation de la HFLAM se bute à une série d'obstacles: ses fondateurs doivent en effet se défendre contre l'image du Juif usurier que l'on dresse devant eux. Leurs difficultés paraissent atteindre un point culminant lorsqu'à propos de cette société, un député fait observer à ses collègues de l'Assemblée législative qu'il «n'a jamais vu un juif prêter sans intérêt ${ }^{3} \ldots$ »

Les pages qui suivent mettent en lumière les efforts des représentants de la HFLAM pour obtenir son incorporation ainsi que la lutte qu'ils engagent contre Shylock, afin de rejeter l'identité sociale qui leur est assignée. Le concept d'identité sociale auquel nous nous référons a été élaboré à travers les travaux de plusieurs disciplines. En sociologie et en histoire, il est notamment au cœur des écrits sur l'orientalisme et le postcolonialisme. Mais son parcours et son utilisation en traversent beaucoup d'autres champs ${ }^{4}$. Les identités sociales sont des représentations symboliques,

2. Désormais MHFLA. Des institutions semblables seront créées par la suite dans plusieurs villes canadiennes, notamment à Vancouver, Toronto, Winnipeg, Calgary et Hamilton.

3. Débats de l'Assemblée législative, texte établi par Maurice Pellerin (Québec, Service de la reconstitution des débats, Bibliothèque de l'Assemblée nationale, 1996), 281-282, 13 législature, $1^{\text {re }}$ session, séances du 5 novembre au 21 décembre 1912, 9 décembre 1912.

4. La définition donnée ici s'appuie plus particulièrement sur l'analyse que Laurence Fontaine fait de ce concept dans une étude des marchands migrants dans la France d'Ancien Régime. Laurence 
construites par des individus ou des groupes et dont ils se servent pour affirmer ou promouvoir leur position sociale, la consolider et la protéger également. Elles peuvent ainsi être revendiquées, imposées ou rejetées, de sorte qu'elles ne sont jamais fixes mais définies et constamment redéfinies dans l'interaction avec d'autres individus ou d'autres groupes ${ }^{5}$. Produits d'une interaction, elles sont aussi des amalgames d'éléments ou de caractéristiques qui résultent d'un choix conscient. Elles ont donc plusieurs facettes, que les individus ou les groupes mettent de l'avant différemment selon leurs interlocuteurs. Cependant tous ne peuvent également se permettre de jouer de ces différentes identités: leur latitude dans ce domaine dépend de leur situation sociale et de leur position de pouvoir.

Dans les sociétés nord-américaines, les minorités ethniques ou ethnoreligieuses offrent un exemple des limites imposées à ces jeux de mise en scène. Que les groupes ou les individus qui en font partie le veuillent ou non, pour les populations issues de l'immigration, l'identité sociale qui se construit dans les rapports avec la population majoritaire ou dominante est souvent et reste longtemps définie en termes ethniques ${ }^{6}$. De fait, la définition de l'identité sociale que nous venons d'énoncer rejoint celle que, depuis le milieu des années 1980, de plus en plus de chercheurs nord-américains ont donné de l'«ethnicité ${ }^{7}$ » (nous emploierons dans le même sens les termes d' «identité ethnique» et d' «identité juive»). Dans cette optique, l'ethnicité apparaît comme un processus de construction à travers lequel les minorités ont, tout au long des $\mathrm{XIX}^{\mathrm{e}}$ et $\mathrm{xx}^{\mathrm{e}}$ siècles, répondu aux pressions à l'assimilation de la population ou de l'ethnoculture dominante, souvent aussi à son hostilité et négocié les conditions de leur inclusion dans la société ${ }^{8}$. Elles ont par ailleurs «réinventé » ou redéfini leur ethnicité selon

Fontaine, Pouvoir, identités et migrations dans les hautes vallées des Alpes occidentales, XVII $-X V I I I^{e}$ siècle (Grenoble, Presses universitaires de Grenoble, 2003), 243 p., chap. VII. Notre réflexion sur les démarches des fondateurs de la HFLAM a notamment été nourrie par la lecture d'un texte préparatoire à cet ouvrage que Mme Fontaine a bien voulu nous faire parvenir. Nous l'en remercions vivement.

5. Ces identités ne sont généralement pas sans lien avec la réalité mais elles en sont distinctes: elles peuvent présenter et interpréter des aspects de cette réalité.

6. Même les Anglais et les Écossais qui, au Canada, dans la première moitié du xx $x^{\mathrm{e}}$ siècle, forment avec les Irlandais les groupes immigrants les plus nombreux, ne font pas nécessairement exception à cette règle.

7. La nature de l'ethnicité a fait l'objet de longs débats parmi les historiens de l'immigration. Voir Journal of American Ethnic History, 12,1 (automne 1992): 3-42 en particulier; l'article de Kathleen Conzen, David Gerber, Ewa Morawska, George Pozzetta et Rudolph Vecoli, «The Invention of Ethnicity: A Perspective from the U.S.A.».

8. Ibid., 4-6, «ethnicity is [...] a process of construction or invention which incorporates, adapts, and amplifies preexisting communal solidarities, cultural attributes, and historical memories». 
le contexte propre à chaque époque et la réalité changeante de leurs dynamiques internes. L'historicité de ce concept est donc fondamentale.

Revenons au cas qui nous intéresse. Dans la société québécoise du début $\mathrm{du} \mathrm{xx}^{\mathrm{e}}$ siècle, une société encore relativement homogène en dépit de sa dualité, chrétienne et, en ce qui concerne la majorité francophone, catholique, les Juifs ont vraisemblablement peu d'occasions d'oublier cet aspect de leur identité. De fait, les événements que nous analysons ici révèlent l'importance du travail qui doit être investi dans la construction symbolique de la minorité juive et combien pour elle les enjeux de cette définition sont cruciaux. Le discours auquel les promoteurs de la HFLAM sont confrontés s'inscrit dans les schèmes de l'antisémitisme qui s'exprime au Québec dans la première moitié $d u x^{e}$ siècle et que des études précédentes nous ont fait connaître déjà̀ ${ }^{9}$ Cependant la création de cet organisme, jamais encore étudiée, l'ensemble des démarches qu'entreprennent ses représentants et les réactions qu'elles provoquent participent de dynamiques plus larges. Elles sont révélatrices des tensions internes de la communauté juive à un moment où celle-ci est transformée par l'afflux de nouveaux immigrants et où l'élite issue de migrations antérieures s'inquiète de préserver ses acquis dans la société montréalaise. Elles mettent en évidence les particularités de la position et du rôle de cette élite. Enfin, elles montrent sous un éclairage nouveau les rapports qui existent entre cette dernière, la population juive récemment immigrée et l'ensemble de la société québécoise à une époque où l'antisémitisme, au Québec comme à travers le monde, commence à se manifester davantage.

Examinons d'abord le contexte très spécifique dans lequel est créée la HFLAM.

\section{UNE COMMUNAUTÉ PLUSIEURS fOIS RECOMPOSÉE}

Les années 1911 et 1912 marquent des sommets dans la plus forte vague d'immigration que le Canada ait connue de toute son histoire ${ }^{10}$. Une

9. Pierre Anctil, Le rendez-vous manqué. Les Juifs de Montréal face au Québec de l'entre-deuxguerres (Québec, Institut québécois de recherche sur la culture, 1988), 366 p. ; Le Devoir, les Juifs et l'immigration: de Bourassa à Laurendeau (Québec, Institut québécois de recherche sur la culture, 1988), 172 p. Voir également Gérard Bouchard, Les deux chanoines: contradiction et ambivalence dans la pensée de Lionel Groulx (Montréal, Boréal, 2003), 313 p. Le terme d'antisémitisme, étymologiquement inexact mais que l'usage a imposé, désigne ici toute forme d'hostilité manifestée envers les Juifs parce que Juifs, quelles que soient ses sources d'inspiration ou la justification qu' on lui donne.

10. On enregistre plus de 331000 entrées en 1911, près de 376000 en 1912. Le sommet absolu — plus de 400000 immigrants - est atteint l'année suivante, avant que cette immigration soit brutalement freinée et presque arrêtée par la Première Guerre mondiale. 
immigration qui, à partir de 1905 surtout, fait de la population juive montréalaise la minorité non britannique la plus importante au Québec. Sa croissance est fulgurante : elle compte 7600 personnes en 1901, près de 30000,10 ans plus tard (si on inclut la région métropolitaine) et plus de 45000 en $1921^{11}$.

Cette population n'avait jamais été homogène et la communauté juive s'était recomposée quelques fois déjà. Les premières familles juives à s'établir officiellement au Québec et à Montréal étaient celles de marchands et commissionnaires qui accompagnaient les armées britanniques, des Ashkénazes, venus d'Angleterre directement ou en passant par les colonies américaines et originaires d'Europe centrale. Puis, au milieu du $\mathrm{xIX}^{\mathrm{e}}$ siècle, quelques dizaines d'autres familles ashkénazes venues d'Angleterre toujours, mais aussi d'Allemagne, d'Alsace et de Pologne s'y établirent à leur tour. À la fin du XIx ${ }^{\mathrm{e}}$ siècle, au moment où les grands mouvements de migration des Juifs d'Europe commençaient à atteindre Montréal, l'une et l'autre composantes de la population juive (qui comptait en tout moins d'un millier de personnes), également anglicisées et presque également prospères, étaient de plus en plus intégrées, liées par des mariages et des associations d'affaires ${ }^{12}$.

Au début $\mathrm{du} \mathrm{xx}^{\mathrm{e}}$ siècle, les synagogues qu'ils ont fondées, la première, de rite sépharade (Shearith Israël, 1768), la seconde, de rite ashkénaze (Sha'ar Hashomayim, 1846), sont celles que fréquentent les gens les plus fortunés et les plus influents. Cela est également vrai du Temple Emanu-El, de tendance réformiste, plus récemment créé $(1882)^{13}$. Toutes ces institutions sont voisines et se trouvent alors établies dans la partie ouest de la ville, anglophone et aisée. Très tôt également, la bourgeoisie juive de Montréal avait mis sur pied, outre ces grandes institutions religieuses, des organismes charitables. Dans la tradition juive, en effet, toutes les formes que prend l'aide apportée à son prochain sont extrêmement valorisées. Le devoir de charité - tsedakah — est commun à tous. Mais c'est aussi en particulier une fonction de l'élite, pour qui les gestes publics de charité sont une source importante de capital social ${ }^{14}$.

11. Gerald Tulchinsky, Taking Root. The Origins of the Canadian Jewish Community (Toronto, Lester Publishing, 1992), 158, 172.

12. Ibid., chap. 1-3.

13. Ibid., 379. Israël Medresh, Le Montréal juif d'autrefois (Sillery, Septentrion, 1997), 60, 263.

14. Gerald Tulchinsky, "Immigration and Charity in the Montreal Jewish Community Before 1890 ", Histoire sociale/Social History, XVI,32, (novembre 1983): 359-380, en particulier 362. Mark Zborowski et Elizabeth Herzog, Life Is with the People: The Culture of the Shtetl (New York, Schoken Books, $4^{e}$ éd. 1964, c.1952), 194-196. 
Depuis la fin du XIX ${ }^{\mathrm{e}}$ siècle, des éléments issus de l'immigration plus récente, celle des années 1870 et 1880, s'étaient joints progressivement à la bourgeoisie juive établie, déjà bien représentée dans le domaine des affaires et certaines professions libérales. Cette nouvelle élite s'affirme en particulier dans l'industrie canadienne du vêtement ${ }^{15}$ et rassemble bientôt les principaux employeurs des milliers de Juifs appauvris qui affluent à Montréal. De ceux-là, Lyon Cohen est le plus connu et le plus puissant sans doute à l'époque qui nous intéresse. Le nom de Harris Vineberg est très souvent mentionné également. Bien entendu, cette élite soutient aussi vigoureusement les œuvres charitables de la communauté et les différentes formes de travail social. C'est elle, désormais, que l'on retrouve à la direction de ses organismes les plus anciens et les plus prestigieux, dont l'Institut Baron de Hirsch (1888). C'est à elle aussi que revient le plus souvent l'initiative d'en créer de nouveaux ${ }^{16}$.

En amenant ici des coreligionnaires pauvres d'origines diverses, la vague migratoire de la fin du XIX ${ }^{\mathrm{e}}$ siècle avait modifié déjà le visage de la petite communauté juive. Elle avait rompu l'équilibre entre Juifs montréalais et nouveaux arrivants et mis à rude épreuve le réseau d'institutions qui existait alors. Celle du début du $\mathrm{xx}^{\mathrm{e}}$ siècle la bouleverse complètement. Un monde sépare les nouveaux venus des Juifs établis issus d'une immigration plus ancienne. La majorité de ceux qui arrivent dans la première décennie $\mathrm{du} \mathrm{xx}{ }^{\mathrm{e}}$ siècle a fui la répression qui suit l'insurrection russe de 1905, à laquelle plusieurs ont participé. Mais ils viennent également de Hongrie, de Pologne et de Roumanie. Ils parlent les langues de leurs pays d'origine mais partagent le yiddish ${ }^{17}$, une langue jugée trop populaire, que les Juifs anglicisés ignorent et méprisent. Ils ont également en commun leur pauvreté: ce sont en majorité des ouvriers ou de petits marchands, précarisés à la fois par les progrès du capitalisme et par les mesures restrictives voire les persécutions dont ils font l'objet, à des degrés divers, dans leurs pays d'origine. À Montréal, ils viennent habiter les vieux quartiers du sud de la ville, près du port et surtout dans l'axe du boulevard Saint-Laurent qu'ils

15. Cette industrie est très fortement concentrée dans les villes de Montréal et de Toronto. Voir Mercedes Steedman, Angels of the Workplace: Women and the Construction of Gender Relations in the Canadian Clothing Industry, 1890-1940 (Toronto, Oxford University Press, 1997), 9.

16. G. Tulchinsky, Taking Root..., op. cit., 136-138.

17. Bien que, dans un premier temps, elles servent souvent de base à la création de sociétés de secours mutuel ou de congrégations religieuses, les origines régionales et nationales diverses de cette population immigrante ne créent pas les divisions les plus significatives. C'est ce dont témoigne entre autres la fondation à Montréal, dès 1907, d'un quotidien de langue yiddish, Der Keneder Adler. 
remontent progressivement vers le nord. En 1912, ils arrivent de plus en plus nombreux dans le quartier Saint-Louis, juste au nord de la rue Sherbrooke ${ }^{18}$. C'est là et dans une moindre mesure dans les quartiers voisins de Saint-Jean-Baptiste et de Laurier, que cette population restera concentrée jusque dans la seconde moitié du siècle. Et c'est dans ce même espace du «couloir» que crée autour de lui le boulevard Saint-Laurent en attirant industries, commerces et main-d'œuvre que travailleront la majorité des nouveaux venus: les Downtowners, par opposition aux Uptowners d'Outremont et des quartiers chics de l'ouest de Montréal. Ils trouvent de l'emploi surtout dans les nombreux ateliers et manufactures de la confection dont, en 1912, ils forment près de $30 \%$ de la force de travail ${ }^{19}$.

Les longues heures et les conditions d'exploitation auxquelles sont soumis les travailleurs du vêtement, déjà révélées par une série d'enquêtes et dénoncées avec véhémence au tournant du siècle, ne se sont guère améliorées depuis ${ }^{20}$. Aussi, dès qu'ils en ont la chance, bon nombre de ces ouvriers tentent de mettre sur pied leurs propres ateliers ou d'ouvrir boutique. Beaucoup de Juifs font en effet de ces petites firmes, pourtant fragiles et instables par définition, une des voies de leur promotion sociale. En 1931, les propriétaires et gérants de firmes formeront près de $30 \%$ de la population active masculine juive du Canada. À Montréal, ce petit patronat juif se concentre plus spécifiquement dans le secteur de la confection $^{21}$.

Chez cette minorité, les divisions de toute nature sont nombreuses. Presque tous les courants idéologiques et religieux qui traversent les populations juives d'Europe s'y trouvent représentés. La lente recomposition de la communauté qui fait suite à l'immigration de masse est marquée par d'innombrables conflits et par des moments de confrontation intenses ${ }^{22}$. De tous les clivages, cependant, le plus fondamental et le plus persistant est la division de classe. Le fait que l'industrie de la confection soit à ce point au cœur de l'économie juive lui donne à cette époque une

18. Sylvie Taschereau, Les détaillants de l'alimentation et les milieux populaires montréalais, thèse de doctorat (histoire), Université du Québec à Montréal, 1992, chap. 3.

19. G. Tulchinsky, Taking Root..., op. cit., 210. Cet historien est également le premier qui ait attiré notre attention sur l'emploi des termes Uptowners et Downtowners chez les Juifs montréalais de cette époque.

20. Ibid., 134. M. Steedman, Angels of the Workplace..., op. cit., 71.

21. Louis Rosenberg, Canada's Jews. A Social and Economic Study of Jews in Canada in the 1930s (Montréal, McGill-Queen’s University Press, 1993, c. 1939), 424 p.

22. I. Medresh, Le Montréal juif d'autrefois, op. cit., et Simon Belkin, Le mouvement ouvrier juif au Canada, 1904-1920 (Sillery, Septentrion, 1999), chap. 15. 
acuité particulière ${ }^{23}$. En effet, plusieurs des membres les plus illustres de l'élite juive montréalaise - ceux que l'on trouve à la tête des grandes institutions charitables et à la direction des comités laïques des plus anciennes et des plus prestigieuses synagogues - comptent parmi les principaux manufacturiers de ce secteur. Or auprès des travailleurs juifs qui sont nombreux à joindre les rangs des syndicats du vêtement, ces manufacturiers ont avant tout la réputation d'être des exploiteurs ${ }^{24}$. Ceux qui, parmi les nouveaux venus, adhèrent aux idéologies de gauche, socialistes surtout mais aussi communistes, anarchistes et bundistes, jouent d'ailleurs un rôle de premier plan dans ces organisations ouvrières ${ }^{25}$.

\section{« PROSPEROUS MEN INSTEAD OF BEGGARS ॥ : CHARITÉ ET DISTANCE SOCIALE}

It is my lifelong ambition to see established in this city a Hebrew Free Loan Association, a society that would help man to help himself; one that would build manhood instead of making paupers, one that would stimulate instead of depress ambition, and in short would make successful and prosperous men instead of beggars ${ }^{26}$.

C'est ainsi que Zigmund Fineberg, l'initiateur et le premier président de la HFLAM, amorce son discours inaugural. Immigrant polonais établi à Montréal en 1887, Fineberg a fait carrière dans le commerce d'abord, puis dans l'immobilier ${ }^{27}$. Homme d'affaires prospère, il ne compte cependant pas à l'époque parmi les figures de proue de la communauté juive. Il parvient néanmoins à associer à son projet plusieurs de ses membres les plus fortunés et les plus influents. Ainsi, la réunion de fondation de cet organisme, tenue en mai 1911, rassemble Lyon Cohen, alors président de la Montreal Clothing Manufacturers' Association, Harris Vineberg et Abraham

23. G. Tulchinsky, Taking Root..., op. cit., 207-208.

24. Harris Vineberg en particulier est connu pour être très dur envers ses employés. I. Medresh, Le Montréal juif d'autrefois, op. cit., 50 et ss. S. Belkin, Le mouvement ouvrier juif..., op. cit., 317.

25. S. Belkin, Le mouvement ouvrier juif..., op. cit., 197; M. Steedman, Angels of the Workplace..., op. cit., chap. 3. Bernard Dansereau, «La place des Juifs dans le mouvement ouvrier québécois au début du $\mathrm{xx}^{\mathrm{e}}$ siècle", dans Pierre Anctil, Ira Robinson et Gérard Bouchard, dir., Juifs et Canadiens français dans la société québécoise (Sillery, Septentrion, 2000), 127-154.

26. Archives nationales du Canada, Fonds de la Hebrew Free loan Association (R4480-0-2-E), Minutes of the Hebrew Free Loan Association, 1911-1927, 2, 28 mai 1911. (Désormais : Minutes of the Hebrew Free Loan Association, 1911-1927).

27. Zigmund Fineberg (1863-1917) fonde en 1905, avec ses fils Joseph et Nathaniel, l'agence immobilière $Z$. Fineberg $\&$ Sons. Il la dirige jusqu'à sa mort, tout comme la MHFLA. Arthur D. Hart, The Jew in Canada (Montréal, Canadian Jewish Publications Limited, 1926), 214. 
Levin $^{28}$, un important manufacturier de galons et ganses. D’autres, parmi les personnes présentes, sont des membres très en vue des professions libérales. C'est le cas de Moses Albert, un pharmacien et l'un des premiers Juifs canadiens à entrer dans cette profession. C'est aussi celui de Samuel William Jacobs. Avocat réputé, Jacobs est déjà l'un des principaux porteparole de la communauté et devient quelques années plus tard un de ses premiers représentants politiques ${ }^{29}$. À ce groupe se joint également Herman Abramovitz, rabbin de la synagogue de Westmount Shaar Hashomayim $^{30}$. Enfin, plusieurs de ses membres fondateurs sont étroitement liés à l'Institut Baron de Hirsch: Harris Vineberg en a été le premier président; S. W. Jacobs en devient président à son tour de 1912 à 1914; $Z$. Fineberg en est longtemps le syndic. Le père de Lyon Cohen, Lazarus, était un de ses administrateurs dans les années $1890^{31}$.

Pour constituer son capital de départ, les membres fondateurs de la HFLAM ont rassemblé, grâce à leurs contributions personnelles, une somme de près de $6000 \$$. Au cours des années qui suivent, cette somme sera augmentée à la fois par les dons et souscriptions de ses membres et par la gestion qu'en font ses administrateurs. Cela permet à l'organisme d'accorder chaque année plusieurs dizaines puis, très vite, plusieurs centaines de prêts dont le montant maximal, de $100 \$$ au moment de la fondation, atteindra $500 \$$ au début des années $1940^{32}$.

En 1883, la Young Men’s Hebrew Benevolent Society ${ }^{33}$ avait quelque temps fait l'essai d'un système de prêts libres d'intérêt, destiné à permettre à des immigrants de se faire colporteurs. Pour des raisons qu' on ignore, cependant - peut-être, suggère Gerald Tulchinsky, par crainte d'encourager une forme de concurrence - cette initiative avait été abandonnée au bout de quelques mois $^{34}$. Les problèmes aigus causés par la récente et formidable vague migratoire, l'ampleur et l'urgence des besoins qu'elle crée, la pauvreté, voire la misère dans laquelle se trouvent plongés par moments

28. G. Tulchinsky, Taking Root..., op. cit., 185.

29. Jacobs est en effet élu député à la Chambre des communes en 1917 et il y siège jusqu’à sa mort en 1938.

30. I. Medresh, Le Montréal juif d'autrefois, op. cit, 60-61.

31. Ibid., 50-51 et A. D. Hart, The Jew in Canada, op. cit., 379.

32. Deux cent cinquante-quatre prêts sont consentis en 1911. On en compte 861 en 1921, dont le montant moyen est de 77 \$. Bibliothèque publique juive de Montréal, Fonds de la Hebrew Free Loan Association, boîte B, Tenth Annual Report, 1921, 9 et "The Hebrew Free Loan Association ", The Montreal Daily Star, 28 mai 1936, 10.

33. Connue à partir de 1891 sous le nom de l'Institut Baron de Hirsch.

34. G. Tulchinsky, «Immigration and Charity...», loc. cit., 374-375. 
les immigrants, travailleurs dans une industrie saisonnière et particulièrement vulnérables à la conjoncture, tout cela a certainement contribué à ce que l'on considère à nouveau cette forme d'aide.

Dans la tradition juive biblique et talmudique, la forme de charité la plus élevée est celle qui permet à son bénéficiaire de garder la tête haute ${ }^{35}$. Dans cette optique, prêter de l'argent est un geste plus noble que faire l'aumône puisqu'il évite à ceux qui le reçoivent d'avoir recours à la charité publique. La HFLAM se réclame de cette tradition. De fait, il existe déjà des sociétés de prêt libre d'intérêt dans les villages et les petites villes de Russie ou d'Europe de l'Est d'où viennent les immigrants ${ }^{36}$. Mais les véritables modèles de cet organisme se trouvent à Londres et surtout à New York ${ }^{37}$. Tout comme la HFLAM, ils ont été mis sur pied dans un contexte de très forte immigration.

Quelle que soit la sincérité du désir d'assistance des fondateurs de la HFLAM et la force du sentiment communautaire ou religieux qui les anime par ailleurs ${ }^{38}$, la création de cette société témoigne certainement aussi d'une préoccupation particulière aux élites de populations minoritaires ou immigrantes. Il s'agit de la crainte que ces coreligionnaires appauvris, nombreux et terriblement visibles, deviennent une charge publique et ternissent en cela, ou par d'autres aspects de leur comportement, leur image et celle d'une communauté qui jusque-là se félicitait d'être bien intégrée et discrète $^{39}$. Sur ce point d'ailleurs, la tradition juive de charité rejoint parfaitement le concept de self-help si fréquemment mis de l'avant par la philanthropie de l'époque et systématiquement rappelé dans les rapports annuels de la HFLAM. L'inquiétude que provoque l'afflux d'immigrants, déjà présente chez l'élite juive vingt ou trente ans plus tôt, se fait plus vive encore.

35. Shelly Tenenbaum, A Credit To Their Community. Jewish Loan Societies in the United States, 18801945 (Detroit, Wayne State University Press, 1987), 32-33.

36. M. Zborowski et E. Herzog, Life Is With The People..., op. cit., 194-213. S. Tenenbaum, A Credit To Their Community..., op. cit., 34.

37. L'importance du modèle new-yorkais ressort très clairement des premiers procès-verbaux du conseil d'administration de la MHFLA. Minutes of the Hebrew Free Loan Association, 1911-1927, 5, 15 juin 1911 et 32, 3 juin 1913.

38. Lyon Cohen est aussi connu pour être capable de gestes d'une grande générosité. Pour lui et pour quelques-uns de ses collègues, ces gestes sont d'autant plus naturels que l'expérience de l'immigration n'est pas si lointaine.

39. G. Tulchinsky, «Immigration and Charity...», loc. cit. Tamara Myers, «The Rise and Fall of Female Jewish Anti-Delinquency Work in Interwar Montreal», dans Bettina Bradbury et Tamara Myers, dir., Negociating Identities in $19^{\text {th }}$ and $20^{\text {th }}$ Century Montreal (Vancouver, University of British Columbia Press, 2005), 175-201. Par ailleurs on retrouve chez les leaders de la communauté italienne de cette époque des préoccupations très semblables. Voir Bruno Ramirez, Les premiers Italiens de Montréal. Les origines de la Petite Italie (Montréal, Boréal Express, 1984) 136 p. 
Elle s'exprime notamment dans le Canadian Jewish Times, journal de la population juive anglophone fondé par Lyon Cohen et Samuel Jacobs où, dès le début du siècle, est publiée une chronique intitulée "As Others See $\mathrm{Us}^{40}{ }^{4}$.

La fondation de la HFLAM survient aussi à un moment où les tensions entre le patronat et les ouvriers du vêtement sont particulièrement intenses. Une grève impliquant les travailleurs d'une manufacture appartenant à Lyon Cohen a eu lieu déjà en 1908; une autre grève a été déclenchée en 1910. Ces mouvements culmineront à l'été de 1912 en une grève générale, dans laquelle les travailleurs juifs se trouveront massivement impliqués. Dans ce contexte, la création d'une société de prêt — qui, au-delà du geste charitable, est présentée aussi comme un outil de promotion économique -, est en même temps une façon de reconnaître les aspirations qu'ont beaucoup de travailleurs juifs à devenir des petits patrons indépendants. En effet, bien que les prêts de la HFLAM soient destinés à venir en aide "à toute personne qui se trouverait temporairement dans le besoin", dans la pratique la plupart seront accordés à de petits détaillants et manufacturiers ou à des hommes et des femmes qui aspirent à le devenir ${ }^{41}$.

C'est également à partir de 1911 que les immigrants juifs commencent à mettre sur pied leurs propres sociétés de prêt. En effet, entre 1911 et 1930, plus d'une centaine de coopératives de crédit sont fondées à Montréal par des Juifs, généralement des travailleurs qualifiés ou de modestes manufacturiers et commerçants ${ }^{42}$. Certaines ne survivent que quelques mois, mais la moitié d'entre elles poursuivront leurs activités pendant plus de 10 ans et, dans plusieurs cas, deux ou trois décennies. Quelques-unes deviendront même des institutions financières considérables, regroupant plusieurs centaines de personnes. Parmi ces coopératives, rares sont celles qui affichent clairement une orientation de gauche. Ce "mouvement» coopératif juif est aussi éclaté que la communauté elle-même: presque toutes les composantes de la population immigrante, en termes politiques, religieux et idéologiques, y participent. Les membres de l'élite ne sont pas en reste: certains d'entre eux encouragent activement la création de ces institutions. C'est notamment le cas de Marcus Sperber, jeune avocat progressiste et membre fondateur lui aussi de la HFLAM ${ }^{43}$. Pour les diri-

40. G. Tulchinsky, Taking Root..., op. cit., 151. Voir également T. Myers, «The Rise and Fall of Female Jewish Anti-Deliquency Work... », loc. cit., 184-187.

41. S. Taschereau, "Les sociétés de prêt juives à Montréal, 1911-1945", Revue d'histoire urbaine/Urban History Review, XXXIII,2 (printemps 2005) : 3-16, en particulier 6. Même modestes, ces prêts peuvent contribuer à mettre sur pied une entreprise ou à la maintenir à flot.

42. S. Taschereau, «Les sociétés de prêt juives...», loc. cit., 3.

43. Sperber parraine ainsi une dizaine de ces sociétés entre 1912 et 1914. 
geants de cette dernière, l'apparition des coopératives est donc probablement une raison supplémentaire d'affirmer leur leadership en matière de promotion économique par le prêt. Mais ils le font bien sûr à la manière d'une élite: la forme que prend cette société et ses règles de fonctionnement soulignent la distance qui les sépare de ceux qu'ils entendent secourir et affirment d'emblée l'autorité qui les rend aptes à juger des qualités morales de leurs bénéficiaires autant que du sérieux de leur demande ${ }^{44}$. Aussi, s'il ne semble pas y avoir d'antagonisme entre la HFLAM et les coopératives, la multiplication rapide de ces dernières montre très explicitement qu'en ce qui concerne cette voie de promotion sociale, les immigrants n'ont pas l'intention de se contenter de l'aide charitable qu'on leur offre.

Ironiquement, au moment où les promoteurs de la HFLAM font face à toutes sortes d'obstacles, la fondation des coopératives de crédit juives passe totalement inaperçue de la population non juive. Contrairement à l'organisme créé par Fineberg et ses collègues, elles sont constituées conformément à une loi québécoise qui existe déjà, bien qu'elle soit toute récente : la Loi des syndicats coopératifs de 1906. La plupart d'entre elles prêtent de l'argent à leurs membres moyennant un intérêt raisonnable, généralement de $6 \%$. Certaines cependant, tout comme la HFLAM, ont pour principe d'accorder des prêts sans réclamer d'intérêt. Ainsi, en 1912, pendant que les fondateurs de cette dernière cherchent le moyen d'obtenir son incorporation, la Papineau Gemelith Chasodim Cooperative voit le jour en toute tranquillité ${ }^{45}$. Son apparition ne provoque aucune réaction particulière de la part des politiciens, non plus que des fonctionnaires chargés de l'enregistrer. Il est vrai que ce nom exotique de Gemelith ou Gemilas Chasodim n'évoque rien pour le greffier de la ville de Montréal où la nouvelle société dépose sa déclaration de fondation ${ }^{46}$.

\section{SHYLOCK ET JUDAS ENTRENT EN SCÈNE : USURE ET ANTISÉMITISME DANS LE QUÉBEC FRANCOPHONE}

Les difficultés de la HFLAM débutent bien avant sa présentation officielle devant le Parlement québécois. Pour obtenir son incorporation, Fineberg et ses collègues amorcent tour à tour, dans les mois qui suivent

44. S. Taschereau, «Les sociétés de prêt juives...», loc. cit., 6 .

45. Archives de la Ville de Montréal, série 120-03-02-00, dossier 14.

46. Ibid. L'orthographe de cette expression qui désigne le prêt libre d'intérêt que l'on fait aux pauvres varie au gré des transcriptions des fonctionnaires municipaux et suivant qu'elle soit écrite en yiddish ou en hébreu. Quant au nom de Papineau, c'est celui d'une rue de l'est de Montréal où l'on trouve une concentration d'immigrants juifs. La Papineau Gemilas Chasodim compte 65 membres au moment de sa fondation. 
sa fondation, deux types de procédure. Celle que suivent la plupart des organismes de charité se limite à des formalités administratives et n'entraîne pas de frais importants: il en coûte $25 \$$ environ ${ }^{47}$. Cependant la forme particulière que prend cette société présente un cas unique, sans précédent légal. Aussi la charte du gouvernement provincial devra plus probablement être accordée par une loi privée, parrainée par un député et votée en chambre $^{48}$. Dans l'un et l'autre cas, leur requête se bute à une forte résistance ${ }^{49}$. Cela tient, semble-t-il, au choix de la raison sociale: le terme "loan», tel qu'il figure dans Hebrew Free Loan Association, n'est pas accepté. Informés du problème, les membres du conseil d'administration proposent alors de rendre plus limpides les objectifs de leur organisme en le nommant plutôt: Hebrew Benevolent Free Loan Association (Association hébraïque de prêt de bienfaisance $)^{50}$. Rien n'y fait. L'objection, apprennentils, vient de haut: c'est le Premier ministre Lomer Gouin lui-même qui bloque la procédure ${ }^{51}$. Pour que la demande d'incorporation soit considérée par l'Assemblée législative, leur fait-on savoir, il faut exclure du nom choisi aussi bien la mention «free», que le mot «loan». Fineberg et ses collaborateurs ne peuvent s'y résoudre ${ }^{52}$. Dès lors, pour obtenir gain de cause, ils mobilisent toutes les ressources dont ils disposent.

D’abord confiée à Maxwell Goldstein, leur conseiller honoraire, cette affaire occupe bientôt plusieurs autres avocats parmi les plus renommés de la communauté juive montréalaise: Peter Bercovitch, proche du gouvernement libéral ${ }^{53}$, puis S. W. Jacobs et Louis Fitch, dont la firme est chargée de présenter le «bill spécial ${ }^{54}$ ». C’est John Thomas Finnie, député libéral de la circonscription de Saint-Laurent, où se trouve concentrée la

47. Minutes of the Hebrew Free Loan Association, 1911-1927, 16, 14 avril 1912.

48. Nous devons à notre collègue Yvan Rousseau ces éclaircissements concernant la démarche d'incorporation.

49. Le procès-verbal de la HFLAM ne précise pas le nom de l'instance gouvernementale en cause. Il s'agit vraisemblablement de l'Inspecteur général des institutions financières.

50. Minutes of the Hebrew Free Loan Association, 1911-1927, 14, 17 décembre 1911.

51. Ibid., 15, 15 février 1912.

52. Ibid., 16, 14 avril 1912.

53. Ibid., 15, 15 février 1912 et 16, 14 avril 1914. Peter Bercovitch est né à Montréal en 1879. Ce sont ses parents qui ont immigré dans cette ville. Son père, Hyman, était marchand tailleur. En 1912, Bercovitch a déjà entamé le volet politique de sa carrière: l'année précédente, il était en effet président du club libéral Laurier. Il sera élu député du quartier Saint-Louis à l'Assemblée législative en 1916 et conservera son siège jusqu'en 1938. En 1940, il deviendra député fédéral et le restera jusqu'à sa mort, deux ans plus tard. Dictionnaire des parlementaires du Québec, 1792-1992 (Sainte-Foy, Les Presses de l'Université Laval, 1992), 58-59.

54. Minutes of the Hebrew Free Loan Association, 1911-1927, 16, 14 avril 1912, et «Needy Hebrew Were Helped», The Gazette, 24 janvier 1913, 4. 
population juive, qui doit parrainer cette future $10 i^{55}$. Finnie a visiblement toutes les difficultés du monde à présenter ce dernier et semble croire un moment que ce soit impossible : c'est ce qu'il laisse entendre à la délégation de la HFLAM venue à Québec discuter stratégie avec lui. Une autre délégation, dont font partie Zigmund Fineberg lui-même et Louis Fitch, se rend elle aussi dans la capitale québécoise présenter les livres de compte de l'association ${ }^{56}$. C'est au prix de cette suite de démarches et de requêtes que la pétition concernant la société de prêt est finalement acceptée par le comité chargé d'examiner les projets de loi, puis officiellement présentée devant la Chambre d'assemblée sous le nom de «Bill 73 ». En fait, il semble bien que de ce point de vue, l'essentiel de la bataille ait été livré avant même qu'ait lieu le débat en chambre.

Les deux premières lectures du Bill 73 se font apparemment sans encombre. Toutefois, lorsque l'Assemblée se forme en comité pour débattre de son adoption, quelques-uns de ses membres expriment leur étonnement et une certaine circonspection face à une société qui affirme vouloir prêter de l'argent sans exiger d'intérêt: s'agit-il d'une société par actions, d'une société d'assurance? Thomas Finnie, le député qui parraine le bill, ainsi que le Premier ministre du Québec, Lomer Gouin, leur fournissent quelques explications. Mais leurs réponses ne satisfont ni le député de Joliette, Joseph-Mathias Tellier, ni le député de Montmagny, Armand Lavergne, et l'un et l'autre ne se gênent pas pour exprimer ouvertement leurs soupçons: «Ce qui va arriver, affirme Tellier, c'est que l'emprunteur qui ne pourra payer n'obtiendra le renouvellement de son emprunt qu'en doublant le capital.» Lavergne, député indépendant et franc-tireur, observe lui aussi qu' "on ne précise pas le montant à prêter». Mais l'essentiel de son commentaire est fait dans un tout autre registre: «Je veux savoir pourquoi les Juifs prêtent sans intérêt. C'est tellement exceptionnel de la part de cette nationalité qu'il doit y avoir quelque chose là-dessous. Je n'ai jamais vu un Juif prêter sans intérêt, à l'exception de Judas Iscarioth qui, d'ailleurs, s'est pendu ${ }^{57}$."

55. John Thomas Finnie (1847-1925) n’est pas juif mais bien écossais de naissance. Médecinexaminateur pour des compagnies d'assurances, il devient député libéral à l'Assemblée législative en 1908. Député de Montréal-Saint-Laurent à partir de 1912, il le reste pendant deux mandats consécutifs. Dictionnaire des parlementaires du Québec..., op. cit., 275.

56. "Needy Hebrew Were Helped», The Gazette, loc. cit.

57. Débats de l'Assemblée législative, texte établi par Maurice Pellerin (Québec, Service de la reconstitution des débats, Bibliothèque de l'Assemblée nationale, 1996), 281-282, 13e législature, $1^{\text {re }}$ session, séances du 5 novembre au 21 décembre 1912, 9 décembre 1912. 
Le lien qu'établit Lavergne entre les Juifs et l'usure a des racines profondes, dans la culture occidentale et chrétienne, qui remontent au Moyen Âge ${ }^{58}$. Il est fréquemment évoqué aussi bien chez les Canadiens d'origine britannique que chez les Canadiens français. Le fait que Shylock, le juif et l'usurier, soit à cette époque une référence commune aux uns et aux autres en témoigne. À travers ce personnage qu'il mettait en scène dans Le marchand de Venise (1600), Sheakespeare opposait chrétiens et juifs, la grâce $d u$ don que savaient respecter les premiers et l'âpreté au gain, la bassesse des seconds ${ }^{59}$. Au début du $x x^{\mathrm{e}}$ siècle, à défaut de connaître Sheakespeare, les lecteurs de la presse à grand tirage du Québec et du Canada anglais connaissent fort bien le nom de Shylock, qui sert à évoquer et à dénoncer des pratiques financières condamnables et la malhonnêteté des marchands ${ }^{60}$.

L'image du Juif usurier est aussi utilisée dans un contexte plus spécifiquement québécois: elle est brandie par moments dans des discours de promotion économique qui s'adressent aux Canadiens français. Celui d'Alphonse Desjardins et de l'abbé Grondin à qui il confie son "catéchisme des Caisses populaires ${ }^{61}$ » et ceux, plus fréquents dans les années 1920 et 1930, de l'Achat-chez-nous, où les Juifs se trouvent associés aux étrangers (Britanniques, Américains et Canadiens anglais) qui dominent l'économie de la province ${ }^{62}$.

Plus généralement, cette figure est un des lieux communs du discours antisémite occidental qui, depuis la fin du XIX ${ }^{\mathrm{e}}$ siècle surtout, à partir de la France et à travers des milieux catholiques et cléricaux ainsi que les écrits d'auteurs tels Édouard Drumont et Charles Maurras, nourrissent les préjugés d'une partie de l'intelligentsia québécoise francophone ${ }^{63}$. Cette

58. Léon Poliakov, Les Juifs et notre histoire (Paris, Flammarion, 1973), 17-22.

59. Selon les règles de la morale chrétienne que les textes de loi de l'Europe catholique continuent d'observer jusque dans la seconde moitié du xvIII ${ }^{\mathrm{e}}$ siècle, le prêt doit être fait dans un esprit d'amitié et aucun intérêt n'est exigible à titre de profit. Il ne peut être conçu que comme une geste de gratitude à l'égard du prêteur. Cela n'empêche pas la pratique du prêt à intérêt, notamment par les premières banques. Cependant, nous dit Bartolomé Clavero, ce n'est pas la justice mais plutôt les obligations sociales de réciprocité qui opèrent alors pour assurer la rétribution du crédit. Bartolomé Clavero, La grâce du don. Anthropologie catholique de l'économie moderne (Paris, Albin Michel, 1991), en particulier 163-175.

60. Voir notamment Henry Trachtenberg, «Ethnic Politics on the Urban Frontier: "Fighting Joe" Martin and the Jews of Winnipeg, 1893-96", Manitoba History, 35, (printemps/été 1998): 214 , en particulier 7.

61. Paul Morency, Alphonse Desjardins et le catéchisme des caisses populaires (Sillery, Septentrion, 2000), 104 et ss, ainsi que Yves Roby, Alphonse Desjardins et les Caisses populaires 1854-1920 (Montréal, Fides, 1964), 43-44, 90.

62. Pierre Anctil, Le rendez-vous manqué..., op. cit., 366.

63. Pierre Anctil, Le Devoir, les Juifs, et l'immigration..., op. cit., chap. 1. 
influence se fait sentir sans que l'on ait besoin d'établir de lien avec le fait juif au Québec et avant même que cette population, qui se concentre essentiellement à Montréal, ne devienne une minorité nombreuse. Ainsi la visibilité, en Europe, de quelques grandes familles juives comme celle des Rothschild contribue sans doute à entretenir ici l'idée du Juif accumulant les richesses, banquier rapace, ou riche prêteur. Pourtant, en 1912, les seuls Juifs qu’a pu rencontrer la majorité des Québécois sont les colporteurs qui, à l'occasion, passent dans leur quartier, dans leur patelin ou devant leur ferme et qu'ils confondent souvent, du reste, avec leurs concurrents libanais ou syriens. S'ils connaissent un usurier, il s'agit presque certainement d'un Canadien français, un marchand rural ou un gros cultivateur dans les campagnes, un prêteur sur gage peut-être, ou même simplement un rentier ou une rentière à la ville. Il est vrai que la présence des Juifs dans le commerce et la très forte proportion de ceux qui tentent leur chance dans ce domaine leur donnent progressivement une grande visibilité à Montréal et, dans une moindre mesure, à Québec et en font les cibles fréquentes de la xénophobie.

La suite du discours de Lavergne est très révélatrice de ses sources d'inspiration. Quitte à prendre quelques libertés avec l'Évangile, en effet, le député de Montmagny passe du même souffle, par une acrobatie déconcertante, de l'image de Shylock à celle de Judas. En somme, de l'usurier et du marchand malhonnête au traître déicide. Ce faisant, Lavergne énonce ce qui, aux yeux de l'Église catholique de l'époque, est l'accusation la plus fondamentale qui puisse être faite aux Juifs: ils sont responsables de la mort du Christ. C'est en cela que son invective porte la marque du milieu auquel il appartient et d'une forme d'antisémitisme particulière aux Canadiens français et aux catholiques et différente de celle que l'on trouve chez les Canadiens d'origine britannique ${ }^{64}$. Elle s'inspire d'un discours élaboré dans les tout premiers temps de l’Église chrétienne et que l’Église

64. L'antisémitisme canadien-français s'exprime souvent de façon plus publique que celui des élites canadiennes-anglaises. Pour être plus discret, ce dernier n'en est pas moins oppressant et ses effets néfastes. Il se fait sentir en particulier dans le secteur anglo-protestant de l'éducation où la présence des Juifs est tolérée mais non pas reconnue, quand elle n'est pas systématiquement restreinte comme ce sera le cas à l'Université McGill dans l'entre-deux-guerres. G. Tulchinsky, Taking Roots..., op. cit., xviii. Voir également Roderick MacLeod et Mary Ann Poutanen, A Meeting of the People: School Boards and Protestant Communities in Quebec, 1801-1998 (Montréal, McGill-Queen's, 2004), chap. 7. Par ailleurs, c'est au cours des années 1930 qu'apparait aussi au Québec un autre discours antisémite, non pas religieux cette fois, mais biologisant et pseudo scientifique, celui que tient Adrien Arcand, fondateur d'un petit parti nazi. Arcand n'est cependant pas proche des milieux nationalistes et trouve plutôt appui au Canada anglais. P. Anctil, Le rendez-vous manqué..., op. cit., 223 et ss. 
catholique romaine reprend au milieu du $\mathrm{xIX}^{\mathrm{e}}$ siècle $^{65}$. Elle trouve un écho chez une partie des élites canadiennes-françaises, surtout dans les cercles ultramontains et nationalistes auxquels appartient Lavergne. Dans l'entredeux-guerres, elle sera exprimée avec plus de virulence encore dans les pages du Devoir et dans des publications plus spécifiquement catholiques. Mais depuis quelques années déjà, les uns et les autres soulignent par moments la présence des Juifs par des commentaires malveillants et des plaisanteries grinçantes ${ }^{66}$. Les événements rapportés ici sont d'ailleurs contemporains d'un épisode assez connu de l'antisémitisme au Québec, dans lequel s'impliquera, pour prendre la défense de l'accusé, le quotidien ultramontain L'Action sociale catholique dont Lavergne est un collaborateur régulier. Il s'agit du procès Plamondon, une poursuite en libelle intentée contre un notaire de la ville de Québec dont les propos haineux ont, en 1910, été à l'origine d'attroupements hostiles et de dommages perpétrés contre des commerces juifs ${ }^{67}$.

Déjà les objections du Premier ministre Gouin et celles de son administration mettaient en cause l'intégrité des dirigeants de la HFLAM aussi clairement que les commentaires faits en chambre par Tellier et Lavergne. Quoique Gouin ne fasse aucune déclaration publique à ce propos ${ }^{68}$, ces objections semblent bien avoir été suggérées sinon par une hostilité, du moins par une méfiance face aux Juifs. Méfiance éveillée et amplifiée peut-être par la suspicion dont font alors l'objet les opérations de prêt. En effet, la condamnation de l'usure est un élément du discours religieux et moral qui, au Québec, reste très présent pendant toute la première moitié du $x^{e}$ siècle. Dans ce contexte, l'idée d'une "free loan association", organisme sans précédent connu au Canada, touche peut-être une sensibilité particulière à l'égard du prêt à intérêt $t^{69}$. Mais bien entendu, elle le fait dans la mesure où elle rejoint un préjugé très répandu à l'égard des Juifs.

65. L. Poliakov, Les Juifs et notre histoire, op. cit., 13-15.

66. P. Anctil, Le Devoir, les Juifs, et l'immigration..., op. cit., chap. 1. G. Tulchinsky, Taking Root..., op. cit., 249-250.

67. The Plamondon Case and S. W. Jacobs, compiled by David Rome (Montreal, Canadian Jewish Archives, 1982), 1:226 p. Voir aussi Jean Hamelin et André Beaulieu, La Presse québécoise: des origines à nos jours (Québec, Les Presses de l’Université Laval, 1973-1990), 5: 1911-1919, 260-263.

68. Nous n'en avons pas trouvé davantage dans sa correspondance et ses dossiers personnels.

69. L'histoire de l'usure et du prêt au Québec reste à faire. Toutefois, il est certain que cette sensibilité face au prêt à intérêt n'est pas spécifique à la société québécoise. Ainsi, aux États-Unis, dans les premières décennies du $\mathrm{xx}^{\mathrm{e}}$ siècle, les efforts de réformateurs pour permettre la création d'institutions de prêt accessibles aux travailleurs et à des gens de conditions modestes se heurtent aux lois sur l'usure qui, dans ce pays, restent d'une extrême sévérité. Voir Lendol Calder, Financing the American Dream: A Cultural History of Consumer Credit (Princeton/Oxford, Princeton University Press, 1999), $377 \mathrm{p}$. 


\section{LES ENJEUX DE LA REPRÉSENTATION}

Quoi qu'il en soit, le Premier ministre se rend pour sa part aux arguments et aux preuves qu'on lui fournit. Mieux, en répondant lui-même aux questions soulevées par les députés, Gouin donne finalement au Bill 73 non seulement son appui mais sa caution. À juger des quelques comptes rendus de presse qui rapportent ce débat, les accusations de Lavergne et de Tellier reçoivent de sa part et de celle de Thomas Finnie des réponses à la fois sobres et mesurées. La discussion ne va pas plus loin. Le Bill 73 est adopté "sur division", mais adopté tout de même, et les amendements qu'on y apporte sont mineurs et sans rapport apparent avec les accusations formulées en chambre. De toute évidence, l'incorporation de cette société n'est pas le principal débat du jour ${ }^{70}$. Peut-être aussi chacun est-il pressé de clore la session et de rentrer chez soi, comme le suggère l'article du Devoir qui en rend compte ${ }^{71}$.

Cela n'empêche pas que l'on fasse encore quelques difficultés aux représentants de la HFLAM en refusant de leur rendre, ainsi que le veut l'habitude ou la règle, le dépôt de 200 \$ qui accompagnait leur pétition. Avant que l'affaire soit définitivement réglée, un an plus tard, il faut à nouveau qu’une délégation formée de Zigmund Fineberg, Max Goldstein, Samuel Jacobs et un certain M. Morgan, rencontre personnellement le Premier ministre Gouin, à l'occasion du passage de ce dernier à Montréal ${ }^{72}$.

La sortie de Lavergne ne semble pas avoir causé beaucoup d'émoi parmi les membres de l'Assemblée législative. Les remarques antisémites ou xénophobes ne font tout simplement pas scandale à l'époque, ni au Québec ni ailleurs dans ce pays. Celles de Lavergne rejoignent les jugements sur les Juifs et plus généralement sur l'immigration que s'autorisent d'autres politiciens ou journalistes canadiens, dans des termes qui ne sont guère plus généreux que ceux rapportés ici ${ }^{73}$. Comme son mentor, Henri

70. Un autre bill présenté par Finnie retient l'attention. Il concerne la question des manuels scolaires et il est l'occasion d'un long affrontement par journaux interposés entre Lomer Gouin, que défend la presse libérale et $\mathrm{M}^{\mathrm{gr}} \mathrm{d}$ 'Amours, le rédacteur de l'Action sociale catholique, défendu dans cette même publication par la plume d'Armand Lavergne. La correspondance et les dossiers de Lomer Gouin qui datent de cette époque ne contiennent rien à propos de la HFLAM. Ils montrent au contraire l'importance que Gouin accorde au débat sur la question scolaire ainsi qu'à ses démêlés avec d'Amours et L'Action sociale catholique. Bibliothèque et Archives nationales du Québec, Fonds Lomer Gouin, (ZC 10/06 4M00 4808), vol. 13, novembre-décembre 1912.

71. "Lettre de Québec», Le Devoir, 1, 10 décembre 1912.

72. «Needy Hebrew Were Helped», The Montreal Gazette, loc. cit.

73. Ainsi en 1907, dans une intervention sur les politiques d'immigration faite à titre de député devant le Parlement d'Ottawa, Lavergne rappelle les propos xénophobes tenus par Frank Oliver avant qu'il devienne ministre de l'Intérieur en 1905 et ceux d'un correspondant du Globe de 
Bourassa $^{74}$, Lavergne s'oppose avec véhémence aux politiques canadiennes qui favorisent une forte immigration. Tous deux la critiquent surtout parce qu'elle menace à leurs yeux l'équilibre des rapports entre les deux "peuples fondateurs" et l'avenir politique des Canadiens français dont ils défendent les droits ${ }^{75}$. Mais, bien sûr, les Juifs sont les étrangers par excellence et le bagage de préjugés qui les concerne les rend particulièrement indésirables comme immigrants et à titre de futurs citoyens. C'est aussi ce qu'il faut lire dans la remarque que fait Lavergne à propos de la HFLAM.

En demandant son incorporation, les dirigeants de cet organisme se présentaient, à la fois face à la communauté juive montréalaise et face à l'ensemble de la société québécoise, comme les membres d'une élite, soucieuse de venir en aide aux démunis. La procédure qu'ils devaient suivre, l'obtention d'un bill privé, amplifiait encore le caractère public de ce geste. Or non seulement la déclaration de Lavergne, faite devant le Parlement, met-elle très publiquement en doute les intentions charitables de Fineberg et celles de ses collaborateurs, mais la réponse qu'on leur fait abolit totalement la distance sociale qu'ils voulaient affirmer et impose également à tous, membres de l'élite ou Juifs ordinaires, une identité particulièrement odieuse. À elle seule en effet, l'accusation d'usure définit une identité: celle d'un être amoral, âpre au gain, prêt pour lui à exploiter son semblable et donc incapable de compassion. Un personnage, en somme, qu'on ne souhaite avoir ni comme voisin ni comme concitoyen $^{76}$.

Toronto. Armand Lavergne, textes choisis par Marc La Terreur (Montréal, Fides, 1968), 28-29. Quelques années plus tôt, Joseph (Joe) Martin, député à l'Assemblée législative du Manitoba, reprochait au ministère de l'Intérieur de ne pas être suffisamment sélectif dans son choix d'immigrants et de laisser trop facilement les Juifs entrer au Canada. Henry Trachtenberg, «Ethnic Politics on the Urban Frontier... ", loc. cit., 6.

74. Lavergne commence sa carrière politique en 1904, en tant que député libéral à la Chambre des communes. C'est un protégé de Laurier mais son parcours et les idées qu'il défend le rapprochent davantage d'Henri Bourassa. En 1903, il fondait avec ce dernier, aux côtés d'Omer Héroux et d'Olivar Asselin, la Ligue nationaliste canadienne. Quelques années plus tard, il devient un collaborateur du Devoir. Franc tireur, refusant de se conformer à une ligne de parti, il est mis à la porte du Parti libéral fédéral en 1907 et passe à la scène politique provinciale en 1908.

75. On doit à Lavergne l'adoption, en 1910, alors qu'il est député à l'Assemblée législative, d'une loi qui rend le français obligatoire dans les services publics québécois. Paul-André Linteau, René Durocher et Jean-Claude Robert, Histoire du Québec contemporain (Montréal, Boréal, 1989), 1: 69.

76. “L'usurier, rappelait l'évêque de Montréal, $\mathrm{M}^{\mathrm{gr}}$ Bourget, dans sa lettre pastorale de 1887 , est $[\ldots]$ un homme inhumain. Car le maudit intérêt qui le domine ferme ses entrailles à tout sentiment de tendresse, d'amour et de compassion. Il est inhumain [...] envers ses voisins, qu'il incommode; envers les pauvres, pour qui il est dur; envers les riches, qu'il cherche à ruiner; envers les veuves et les orphelins, qu'il trouve moyen de dépouiller; envers ses concitoyens, qu'il sait exproprier habilement, et qui iront arroser de leurs larmes une terre étrangère [...]». P. Morency, Alphonse Desjardins et le catéchisme des Caisses populaires, op. cit., 115. 
Si les Uptowners craignaient que leur image ne soit ternie par l'arrivée de leurs coreligionnaires immigrants, ces événements confirment à quel point sur ce plan, leurs acquis sont fragiles et leur identité irrémédiablement liée à celle des nouveaux venus. Mais bien sûr, leur position en tant qu'élite n'est pas seule en cause. Les implications du commentaire de Lavergne sont plus graves. En quelques mots seulement, celui-ci a symboliquement bien sûr, mais sans appel — exclu les Juifs des limites de la société québécoise et canadienne: "c'est tellement inhabituel de la part de cette nationalité...». Faire allusion à Shylock, puis évoquer de surcroît Judas Iscarioth, figure ultime du traitre dans le monde chrétien et catholique, c'est rejeter de façon plus définitive encore, si cela est possible, toute prétention qu'ont les Juifs à être reconnus comme d'honnêtes citoyens de ce pays. Sur ce point, les propos de Lavergne ne sont pas sans rappeler une déclaration faite par Henri Bourassa devant le Parlement canadien quelques années plus tôt et selon laquelle «les Juifs ne s'assimilent pas à la population où ils vivent. Ils ne deviennent citoyens d'un pays qu'... [afin] de s'enrichir tout en contribuant le moins possible au progrès du pays ${ }^{77}$."

À juger du contenu des principaux journaux de Montréal et de Québec, l'intervention de Lavergne passe pratiquement inaperçue des lecteurs canadiens-français et canadiens-anglais. Ainsi, la version du débat que rapporte Le Devoir est «épurée » de ses références bibliques et de ses accusations. Par contre, la réaction des députés est immédiatement relevée par le Canadian Jewish Times que dirige depuis peu Marcus Sperber. Le commentaire qu'on y trouve est aussi subtil que l'attaque était fielleuse et il est mordant à souhait :

The Quebec Legislature for the first time in its existence saw the spectacle of what appeared to be unique - Jews asking to be permitted to loan money without interest. It seemed like a streak from the millenium. They were surprised, and rightly so, for their surprise came from suspicion. And the suspicion was justifiable. Too often they had been asked by a Gentile association for a charter to do business as a philanthropic society or as an art society, when these organizations cloaked a money getting scheme, a lottery or even a

77. Cité par Pierre Anctil, Le Devoir, les Juifs, et l'immigration..., op. cit., 38-39. Il convient de rappeler ici qu'au cours des décennies qui suivent, après avoir quitté la scène politique pour se concentrer sur son rôle de directeur du Devoir, ce leader nationaliste, s'appuyant sur sa foi chrétienne et son sens de la justice, rejettera ses positions antisémites. En plus d'une occasion, il prendra aussi la défense des Juifs, notamment au cours des années 1930, au moment où cette population sera particulièrement vulnérable. 
loan-shark body. Gentiles of a questionable sort raised their suspicions. It remained for Jews to set them aside ${ }^{78}$.

Le ton de cette réplique dit assez combien l'insulte est cuisante et combien on est sensible à l'accusation de pratiquer l'usure. La colère y est palpable et donne le pouls de l'élite de la communauté juive. Mais précisément, comme il est publié en anglais, ce journal ne peut s'adresser à l'époque qu'à une minorité de Juifs anglicisés. Les immigrants récents, eux, lisent plutôt le quotidien yiddish Der Keneder Odler, lequel reste muet sur cette affaire, peut-être parce qu'à cette époque, il n'a pas de correspondant régulier à Québec. De plus, ce petit article ne circule vraisemblablement pas au-delà des limites de la population juive. Il est peu probable qu'on se serait permis autrement de retourner de façon aussi directe l'insulte à l'offenseur en évoquant ainsi des "Gentils d'un genre peu recommandable». Ce texte indique bien cependant que les dirigeants de la HFLAM n'entendent pas accepter sans riposte le miroir que l'on prétend leur tendre et qu'à ce jeu de représentations ils ne seront pas de reste. Lorsqu'ils décident de s'adresser à l'ensemble du lectorat montréalais, ils choisissent toutefois d'autres journaux et d'autres termes.

\section{"L'AUTRE SOIR, UN HOMME ENTRAIT DANS LE BUREAU... " : NÉGOCIER L'IDENTITÉ JUIVE}

Moins d'un mois après avoir obtenu sa charte provinciale, en janvier 1913 et une fois passée, peut-être, la première réaction de colère, la HFLAM entreprend en effet une campagne de presse. Deux articles paraissent en même temps dans The Gazette et La Patrie ${ }^{79}$. Le Devoir leur emboîte le pas quelques jours plus tard. Tous ces textes sont basés sur un communiqué préparé par la HFLAM. L'introduction de l'article du Devoir mérite d'être retranscrite ici, car elle montre bien le sens de la démarche entreprise: "L'autre soir, un homme d'une soixantaine d'années entrait dans le bureau, disant à l'un de nos collaborateurs: je suis juif et je m'intéresse à la Hebrew Free Loan Association. On en a un peu parlé dans les journaux. Voici le résultat des opérations des seize derniers mois. Lisez-le [...] peutêtre trouverez-vous qu'il vaudrait la peine de fonder chez les chrétiens une association similaire ${ }^{80} »$ ?

78. The Canadian Jewish Times, 13 décembre 1912, 12. Nos italiques.

79. "Needy Hebrew Were Helped», The Montreal Gazette, loc. cit.; "Comment les Juifs se protègent", La Patrie, 24 janvier 1913, 1.

80. «L'exemple des Juifs», Le Devoir, 29 janvier 1913, 1. 
Le fait de signaler dans les journaux non juifs la création de la HFLAM est une politique commune aux institutions semblables qui existent aux États-Unis ${ }^{81}$. En effet, d'après leurs statuts, ces sociétés offrent leur aide à toute personne qui se trouverait temporairement dans le besoin, «sans égard à sa religion ou à sa "race" ", ce que fait certainement la société montréalaise depuis ses débuts ${ }^{82}$. Mais visiblement, le but des articles mentionnés ici n'est pas tant d'inviter les chrétiens à se prévaloir de son aide que de mettre en valeur cette institution, l'esprit qui l'anime et bien sûr, à travers eux, valoriser l'ensemble de la communauté juive ${ }^{83}$.

A priori, la réaction des trois journaux concernés est unanimement bienveillante. Le Devoir, sous la plume d'Omer Héroux, salue le «magnifique esprit d'organisation et d'entraide qui règne chez les Juifs de Montréal» - un commentaire un peu ambivalent qui, chez ce journaliste, prendra en d'autres temps l'allure d'une accusation ${ }^{84}$. Mais il accueille ici favorablement l'idée de fonder une institution semblable chez les chrétiens. Seule The Gazette, cependant, rend vraiment compte des difficultés rencontrées par la société de prêt juive et insiste sur le fait qu'elle soit ouverte aux non-Juifs. À ce chapitre, La Patrie reste silencieuse et Le Devoir ouvertement sceptique. De même, l'invitation faite aux journalistes d'assister à l'une ou l'autre des réunions du conseil d'administration n'apparaît pas dans les journaux français. Craint-on d'encourager ces contacts? Ce renseignement leur a-t-il été communiqué? Il est certain que la façon dont l'information est rendue par les uns et les autres trahit des perceptions et des rapports différents entre l'élite juive et les principaux quotidiens français de Montréal, d'une part, et entre elle et certains des journaux anglais de cette ville, d'autre part. Ces divergences tiennent en partie à la plus grande familiarité que les membres de la bourgeoisie juive ont avec la culture et la société anglo-

81. S. Tenenbaum, A Credit To Their Community..., op. cit., 90-92.

82. Les documents que nous avons pu consulter jusqu'à présent ne permettent pas de connaître la proportion que ces prêts représentent par rapport à l'ensemble. En 1914, cependant, environ $15 \%$ des bénéficiaires de la New York Hebrew Free Loan Society étaient des non-Juifs. S. Tenenbaum, A Credit To Their Community..., op. cit., 92.

83. En fait, plusieurs années plus tard, au moment où les HFLA d'Amérique du Nord débattent du maintien de leur politique de prêt aux non-Juifs, Nathaliel, le fils de Z. Fineberg et son successeur à la tête de la HFLAM, explique à son vis-à-vis de San Franciso que sa société a très vite abandonné pour sa part la politique de publicité dans les journaux non juifs pour éviter de se trouver dans des situations embarrassantes et susceptibles d'entretenir l'image de l'usurier juif. S. Tenenbaum, A Credit to Their Community..., op. cit., 90-91.

84. P. Anctil, Le Devoir, les Juifs, et l'immigration..., op. cit., 83-85. 
saxonnes et reflètent également la façon très différente dont les réticences à l'égard des Juifs se manifestent chez les uns et les autres ${ }^{85}$.

Quel que soit leur interlocuteur cependant, les fondateurs de la HFLAM ne peuvent se permettre d'ignorer l'accusation d'usure qui leur a été faite, non plus que la référence implicite à Shylock. Pour prévenir ou désamorcer des réactions du type de celle de Lavergne, ils choisissent au contraire de se porter, preuve à l'appui, au-devant des soupçons. Ils mettent également beaucoup de soin à décomposer l'image de Shylock, à rompre le lien entre le Juif et l'usurier qu'avait renoué le discours de Lavergne et à reconstruire une identité juive à laquelle sont associées des qualités dignes d'admiration et d'émulation. Des qualités de civisme aussi, preuve de leur valeur en tant que citoyens. Ainsi, bien que la lutte contre l'usure ne soit pas en réalité leur préoccupation première ${ }^{86}$, les articles du Devoir, de La Patrie et de The Gazette la mentionnent tous expressément et reprennent le même passage où l'on utilise une terminologie familière aussi bien aux lecteurs canadiens-français que canadiens-anglais, mais ethniquement neutre cependant: "There are a great many who, through the assistance of this Association, have been prevented from falling into the hands of loan sharks, whilst others have been assisted to free themselves from the clutches of these people», précise The Gazette $e^{87}$; «Par elle, dit la notice, beaucoup de gens ont été arrachés aux serres des usuriers...», reprend Le Devoir $^{88}$. Pour mieux échapper à Shylock, en somme, les dirigeants de la HFLAM doivent remettre en scène le personnage de l'usurier avant de le mettre à distance («these people») et de redéfinir en la déplaçant la frontière entre «nous» et «eux».

En effet, dire que la création d'une institution semblable à la HFLAM pourrait rendre quelques services aux chrétiens, c'est suggérer, de façon plus délicate mais non moins explicite que ne le faisait le Canadian Jewish Times, qu'il se trouve également des usuriers parmi les Gentils. Ces individus, contre lesquels se dresse la société de prêt, représentent un ennemi commun qui, dès lors, rassemble Juifs et non-Juifs en un «nous» élargi. Les

85. P. Anctil, Le rendez-vous manqué..., op. cit., et G. Tulchinsky, Taking Root..., op. cit.

86. Les procès-verbaux établis au moment de la fondation de la HFLA et au cours de sa première année d'existence n'en font pas mention. Par contre, en octobre 1912, le conseil d'administration discute du cas d'un usurier qui sévit vraisemblablement à l'intérieur de la communauté juive. Il est alors convenu de lui faire parvenir une lettre pour le dissuader de poursuivre de telles pratiques. Minutes of the Hebrew Free Loan Association, 1911-1927, 24-25, 28 octobre 1912.

87. The Gazette, «Needy Hebrew Were Helped», loc. cit.

88. Le Devoir, «L'exemple des Juifs», loc. cit. 
communiqués de cet organisme et le geste par lequel ils sont présentés à la presse anglaise et française sont une invitation à la collaboration entre chrétiens et juifs et affirment d'une façon qui ne laisse place à aucune équivoque le souci qu'ont ces derniers de contribuer positivement à la société québécoise.

Dans ce sens, la campagne de presse de la HFLAM s'apparente à plusieurs des interventions faites par les représentants de l'élite juive au cours de la première moitié $\mathrm{du} \mathrm{xx}^{\mathrm{e}}$ siècle dans le but de défendre la réputation de leur communauté et les droits de citoyenneté de ses membres. Ils le font à travers les journaux - outils privilégiés, où se façonne constamment l'image de l'«autre», en particulier celle de l'«étranger»mais aussi en s'adressant directement à leurs responsables et en cherchant l'appui de journalistes tolérants. C'est ainsi qu'en 1912, Samuel Jacobs, heurté et inquiet de voir les Juifs représentés systématiquement de façon négative dans les pages du Devoir, engage à ce propos une correspondance avec son directeur, Henri Bourassa ${ }^{89}$. De même, dans les années 1930, à une époque où l'antisémitisme se fera particulièrement intense, Hannaniah Meir Caiserman, secrétaire général du Congrès juif canadien, adoptera une politique d'intervention publique et systématique dans bon nombre de journaux et auprès de plusieurs journalistes ${ }^{90}$.

La démarche de la HFLAM se distingue néanmoins de ces exemples sur quelques points importants: il n'y a pas ici d'affrontement direct. On ne prend pas à partie les députés, ce qui serait délicat et risqué, compte tenu de la réaction première de Lomer Gouin lui-même. Les fondateurs de cet organisme ne peuvent par ailleurs en appeler de la rationalité et du sens de la justice du public dans les termes où Jacobs le fait lorsqu'il interpelle Bourassa $^{91}$. Enfin bien que cette campagne de presse soit de fait une revendication d'inclusion dans les sociétés québécoise et canadienne, c'est une revendication indirecte et prudente, fort différente de celles que Caiserman fera dans les années 1930, où il insistera nommément sur les droits des Juifs en tant que citoyens canadiens. Mais en 1912, la population juive est fragilisée de ce point de vue par sa forte proportion d'immigrants récents. «Tous les groupes sociaux, observe Laurence Fontaine, ne sont pas

89. P. Anctil, Le Devoir, les Juifs, et l'immigration..., op. cit., 41-43.

90. P. Anctil, Le rendez-vous manqué..., op. cit., chap. 5.

91. "Croyez-vous servir un objectif utile en insistant sur la foi religieuse de tout Juif coupable d'un manquement mineur à un règlement civil? [...] Vous qui faites preuve de civisme et proclamez avoir à cœur l'intérêt de ce pays, approuvez-vous de telles méthodes", écrivait notamment Jacobs. P. Anctil, Le Devoir, les Juifs et l'immigration..., op. cit., 41. 
également vulnérables aux manipulations de leur identité. La reconnaissance par l'ensemble du corps social de certains statuts limite les manipulations possibles ${ }^{92}$.» Or sur ce plan, l'acquisition de la citoyenneté canadienne représente certainement une importante garantie juridique, bien qu'elle ne se soit pas toujours avérée une protection suffisante pour les nouveaux venus $^{93}$. Des événements comme ceux qui sont rapportés ici ne peuvent qu'inciter davantage les Juifs à adopter cette citoyenneté, ce qu'ils font très rapidement ${ }^{94}$, et à élire des représentants politiques à tous les niveaux de gouvernement, ce qu'ils parviennent à faire également entre 1912 et 1916. Cela ne les mettra pas à l'abri de l'antisémitisme, mais leur fournira de meilleurs outils de négociation, obligeant sans doute les autres députés et conseillers municipaux à plus de retenue et donnant plus d'assurance aux répliques qu'ils feront dans la presse. Entre-temps, les porte-parole de la HFLAM avancent sur un terrain délicat. L'image du Juif usurier qu'ils cherchent à contrer a presque valeur d'archétype tant son ancrage est profond et ils sont conscients du fait que l'institution qu'ils dirigent lui est particulièrement vulnérable.

La fréquence de ce type d'intervention au cours de la première moitié $\mathrm{du} \mathrm{xx}^{\mathrm{e}}$ siècle nous donne une mesure de l'énergie que les leaders de la communauté juive montréalaise sont contraints d'investir alors, face aux autorités et face à l'ensemble la population québécoise, dans un travail constant de "présentation de soi ${ }^{95}$ ", de définition d'une identité juive aussi englobante que celle que leur assignent les discours antisémites. Les démarches des représentants de la HFLAM révèlent pourtant d'autres aspects des rapports qui s'établissent entre l'élite de la population juive et différentes composantes de la société québécoise.

\section{JUIFS, CITOYENS OU COLLÈGUES : LES VISAGES D’UNE ÉLITE}

Avant qu'ils n'engagent la lutte sur le terrain symbolique, du moins avant qu'ils ne l'engagent à travers les journaux, Fineberg et ses collaborateurs mobilisent en effet leurs ressources pour obtenir l'incorporation de la

92. L. Fontaine, Pouvoirs, identités et migrations..., op. cit., 149-150.

93. Voir Barbara Roberts, Whence They Came. Deportation From Canada, 1900-1935 (Ottawa, Les Presses de l'Université d'Ottawa, 1997, c.1988), 246 p.

94. En 1931, presque $80 \%$ des Juifs résidants au Québec se déclarent citoyens canadiens, alors que $45 \%$ d'entre eux environ sont nés au Canada. P. Anctil, Le rendez-vous manqué..., op. cit., 40 .

95. L. Fontaine, Pouvoir, identités et migrations..., op. cit.,150. Cette historienne reprend ici un concept de Pierre Bourdieu. Voir La distinction. Critique sociale du jugement (Paris, Les Éditions de Minuit, 1979), 554-564. 
nouvelle institution de prêt. Or ces ressources et les relations qu'ils entretiennent dans différents milieux de la société montréalaise et québécoise leur permettent de dénouer l'impasse et de négocier une solution «au sommet» avec les membres les plus influents du gouvernement. Elles sont le fait d'une élite bien insérée dans des réseaux professionnels anglophones et francophones et qui a aussi des liens avec les milieux politiques québécois. Ainsi, ce monsieur Morgan, qui participe en deux occasions au moins aux démarches que font les porte-parole de la HFLAM à Québec et auprès du Premier ministre Gouin, est un membre de la famille propriétaire du grand magasin à rayons montréalais Henry Morgan $\& \mathrm{Co}^{96}$. Max Goldstein, pour sa part, est né et a grandi dans la ville de Québec. Au cours de sa carrière, il s'est associé à des Canadiens français aussi bien qu'à des Canadiens anglais ${ }^{97}$. Les avocats qui, par la suite, représentent la HFLAM ont fait leurs études non seulement à l'Université McGill, mais aussi à l'Université Laval: Bercovitch et Jacobs ont fréquenté ces deux institutions. Ils ont des entrées au Parti libéral et de toute évidence s'en servent, de même qu'ils prennent appui sur les milieux d'affaires. Comme celles de plusieurs de leurs contemporains, en effet, leurs carrières chevauchent tous ces domaines ${ }^{98}$.

Le procès Plamondon que nous évoquions plus tôt offre lui aussi un bon exemple de ces liens. Ce procès s'ouvre à Québec en 1913, quelques mois à peine après l'incorporation de la HFLAM. Samuel W. Jacobs y figure au tout premier plan, en tant qu'avocat de la poursuite. Or lorsque Jacobs présente la preuve de ce procès, il est en mesure de citer, pour défendre la réputation des Juifs, une lettre très chaleureuse que lui a adressée personnellement, quelques mois auparavant, le doyen de la Faculté de droit de l'Université Laval, le juge Mathieu ${ }^{99}$. Jacobs entretient par ailleurs, depuis 1910 au moins, des rapports professionnels avec Louis-Alexandre

96. Il s'agit peut-être en fait du président de cette compagnie, James Morgan. En 1912, au moment où la HFLAM s'établit dans de nouveaux locaux, rue Ontario, celui-ci offre gracieusement le nouveau mobilier de la salle du conseil d'administration. Bibliothèque publique juive de Montréal, Fonds de la Hebrew Free Loan Association, Annual Report of the Hebrew Free Loan Association, 1939, 16. En 1921, James Morgan figure toujours parmi les principaux donateurs de cette société.

97. C’est d'ailleurs en français que Pierre Beullac, un de ses anciens associés, fait paraître sa biographie, à laquelle il ajoute une appréciation amicale, dans un ouvrage commémoratif bilingue, Le centenaire du barreau de Montréal, 1849-1949 (Montréal, Librairie Ducharme, 1949), 166167.

98. Dans les années 1920, l'un et l'autre dirigent d'importantes compagnies d'assurances.

99. D. Rome, The Plamondon Case, 2: 144-145. 
Taschereau, ministre des Travaux publics et du Travail dans le gouvernement Gouin et futur Premier ministre de la province ${ }^{100}$.

En fait, sur ce plan, les négociations qui entourent la naissance de la HFLAM ne constituent pas un cas isolé, mais semblent être plutôt un élément récurrent de l'histoire de la communauté juive canadienne au moins jusqu'au lendemain de la Deuxième Guerre mondiale. Plusieurs des épisodes d'antisémitisme qui nous sont connus à ce jour ont en effet donné lieu à des démarches similaires. Le cas le plus dramatique et le plus navrant est celui des efforts, restés vains, menés en 1939 auprès du gouvernement canadien pour tenter d'infléchir sa décision concernant l'accueil de réfugiés juifs, dans un contexte de xénophobie et d'antisémitisme prononcés ${ }^{101}$. Hirsch Wolofsky, le fondateur du Keneder Odler, mentionne dans ses Mémoires des pressions faites dans le même but et avec plus de succès quelques années plus tôt ${ }^{102}$. Quelle qu'ait été chaque fois l'issue de ces démarches, elles laissent entrevoir, au-delà des individus qui y sont impliqués et sur lesquels l'attention des chercheurs s'est jusqu'à présent trop exclusivement concentrée - ou que peut-être nous avons eu tendance à trop systématiquement ethniciser - l'interpénétration de réseaux sociaux juifs, canadiens-français et canadiens-anglais. Or nous savons très peu de choses de ces relations que des moments de tension ou de négociation intenses peuvent nous révéler, mais qui en réalité se tissent dans le long terme et à travers des contacts fréquents. Là pourtant se trouvent quelquesunes des clefs dont nous avons besoin pour saisir dans leur complexité et leurs contradictions les rapports qui se sont établis entre la minorité juive et les Québécois d'origine française ou britannique et qui expliquent aussi la progression apparemment rapide de l'intégration des Juifs dans la seconde moitié du $\mathrm{xx}^{\mathrm{e}}$ siècle.

Le contraste souligné au début de cet article entre l'expérience de la HFLAM et celles des coopératives qui naissent au même moment paraît paradoxal et il est ironique à plus d'un titre. Les préoccupations, voire les inquiétudes des autorités québécoises ou canadiennes ont habituellement pour effet d'attirer l'attention sur les immigrants ordinaires plutôt que leurs

100. Jacques Lamarche, Louis-Alexandre Taschereau (Montréal, Lidec, 1997), 19. Taschereau, dont Bercovitch deviendra un proche conseiller dans les années 1920 et 1930, est en effet un des avocats consultés en préparation de ce procès.

101. Irving Abella et Harold Troper, None Is Too Many: Canada and the Jews of Europe 1933-1948 (Toronto, Key Porter, $3^{\mathrm{e}}$ édition, 2000), $340 \mathrm{p}$.

102. Hirsch Wolofsky, Mayn Lebns Rayze. Un demi-siècle de vie yiddish à Montréal (Sillery, Septentrion, 2000), $394 \mathrm{p}$. 
élites ${ }^{103}$. Manifestement, bien que la plupart soient enregistrées sous le nom générique de «loan association» ou de "loan society», les coopératives juives échappent à cette règle. Cela, nous l'avons dit, tient en partie au fait qu’elles sont créées conformément à une loi québécoise qui existe déjà et limite les démarches nécessaires à leur incorporation à l'enregistrement d'un formulaire réglementaire. Dès lors, leur fondation est déclarée dans la quiétude du bureau du greffier, un espace public mais somme toute discret et, en ce qui concerne les coopératives de prêt libre d'intérêt (gemilas chasodim), dans l'anonymat que confère de surcroît une langue étrangère. Par contraste, la démarche administrative à laquelle doit se conformer la HFLAM et qu'elle entreprend aussi à titre d'élite, ne permet pas cet anonymat.

\section{CONCLUSION}

En dépit de sa position de classe (qu'elle met en évidence lorsqu'elle s'adresse à sa communauté), l'élite juive montréalaise reste vulnérable à la fois parce qu'elle est l'élite d'une population immigrante, non chrétienne de surcroît et parce qu'elle est juive : à cette époque, l'antisémitisme est une composante de la culture québécoise catholique et une composante active de la culture chrétienne occidentale. Aussi, quelle que soit la distance culturelle et sociale qui la sépare des Juifs ordinaires, il ne lui est pas permis de s'en distinguer. Et comme cela devient évident au moment de l'immigration de masse, elle est consciente du fait que ses interventions pour défendre et imposer une identité juive positive sont une des conditions de sa participation à cette société et de la participation de ses coreligionnaires en tant que citoyens. À titre individuel ou privé cependant, les membres de cette élite sont moins strictement définis par leur ethnicité et jouent d'un registre d'identités plus large: de fait, les promoteurs de la HFLAM peuvent se présenter en tant que membres estimés d'une communauté de gens d'affaires (ce dont $\mathrm{M}$. Morgan se porte garant) ou, grâce à messieurs Jacobs et Bercovitch, comme membres d'un corps professionnel fort bien représenté parmi les politiciens québécois et membres également du réseau politique libéral. En somme, ils agissent simultanément sur différents terrains, avec différentes armes. Les démarches qu'ils entreprennent, évidemment hors de portée des gens ordinaires, s'appuient sur des relations nouées dans des milieux variés et influents.

103. C'est ce qui se produit lorsqu'elles s'inquiètent de la délinquance juvénile. T. Myers, «The Rise and Fall of Female Jewish Anti-Delinquency Work», loc. cit. 
L'historiographie de l'immigration a jusqu'à présent fait une large part à l'étude de la xénophobie, du racisme et de l'antisémitisme sous toutes ses formes ${ }^{104}$. Les obstacles que rencontrent les représentants de la HFLAM doivent certainement être vus sous cet angle, car ils signalent à nouveau la force des préjugés qu'on entretient à l'égard des Juifs dans le Québec d'avant la Deuxième Guerre mondiale ${ }^{105}$. Le plus souvent, cependant, ces attitudes et ces gestes d'hostilité ont coexisté avec divers types de contact et d'échange. Elles ne représentent en somme qu'une partie des rapports qui s'engagent entre une population immigrée et la population majoritaire ou dominante. Mais tandis que des travaux, relativement nombreux, se sont préoccupés de la dynamique des rapports internes de la communauté juive et nous en ont rendu un portrait de plus en plus complexe et fascinant, les relations qu'elle a entretenues avec le reste de la société québécoise et canadienne paraissent toujours d'une étonnante uniformité ${ }^{106}$. Peu de choses en effet se sont ajoutées au constat d'un "rendez-vous manqué» que Pierre Anctil faisait il y a 15 ans $^{107}$, au regard de la suite d'incidents et de confrontations qui marquent les rapports entre les politiciens et leaders québécois et les représentants de cette communauté. Pourtant ce bilan, qui met l'accent sur l'isolement des Juifs, reste incomplet. Cette population n'a pas vécu et évolué "parallèlement» à la société qui l'entoure. Il s'agit d'une population nombreuse qui, en dépit de sa concentration dans la ville, côtoie et entre constamment en contact avec des non-Juifs. Une population qui joue un rôle significatif, voire de premier plan dans d'importants secteurs de l'économie de la ville ${ }^{108}$, qui très vite aussi est bien représentée parmi les membres des professions libérales et présente dans

104. Sylvie Taschereau, «Migrations et relations ethniques», dans Denise Lemieux, dir. et al., Traité de la culture. Le Québec. Son patrimoine. Ses modes de vie et ses productions culturelles (Québec, Les Presse de l'Université Laval/Les Éditions de l'IQRC, 2003), 201-218. En ce qui concerne le Canada, voir Roberto Perin, "Writing About Ethnicity», dans John Shultz dir., Writing About Canada: A Handbook for Modern Canadian History (Scarborough, Prentice-Hall, 1990), 201-230.

105. Outre les ouvrages de P. Anctil mentionnés précédemment, voir Gary Caldwell, "L’antisémitisme au Québec», dans Pierre Anctil et Gary Caldwell, dir., Juifs et réalités juives du Québec (Québec, Institut québécois de recherche sur la culture, 1984), 371 p. L’analyse qu'Esther Delisle fait de ce phénomène est plus discutable. Son ouvrage présente cependant de très nombreux exemples du discours antisémite que l'on trouve au Québec dans la première moitié du $\mathrm{xx}^{\mathrm{e}}$ siècle: Esther Delisle, Le traître et le Juif. Lionel Groulx, Le Devoir et le délire du nationalisme d'extrême droite dans la province de Québec (Outremont, les Éditions de l'Étincelle, 1992), 284 p.

106. S. Taschereau, "Migrations et relations ethniques», loc. cit.

107. P. Anctil, Le rendez-vous manqué..., op. cit. Voir aussi Jacques Langlais et David Rome, Juifs et Québécois français. 200 ans d'histoire commune (Montréal, Fides, 1986), 286 p.

108. Dans celui de la confection mais aussi, ce qui est moins connu, celui de l'industrie et de la distribution alimentaire. S. Taschereau, Les petits détaillants..., op. cit., chap. 2. 
plusieurs des principales institutions de la société québécoise. Pourtant, bien que l'on reconnaisse maintenant la part active qu'ont prise les Juifs dans le mouvement ouvrier montréalais et canadien dès le tournant du $\mathrm{xx}^{\mathrm{e}}$ siècle ${ }^{109}$, la plupart de ces activités ne sont pas considérées sous l'angle des rapports entre minorité et majorité et nous ne savons rien des aménagements ou des transformations mutuelles auxquels elles ont pu donner lieu.

109. B. Dansereau, «La place des Juifs dans le mouvement ouvrier québécois», loc. cit. 